\section{Analysis of process factors of dry fermented salami to control Listeria monocytogenes}

\author{
Enrico Novelli, ${ }^{1}$ Lucia Dal Santo, ${ }^{2}$ \\ Stefania Balzan, ${ }^{1}$ Barbara Cardazzo, \\ Dino Spolaor, ${ }^{2}$ Angiolella Lombardi, ${ }^{2}$ \\ Lisa Carraro, ${ }^{1}$ Luca Fasolato ${ }^{1}$ \\ ${ }^{1}$ Department of Comparative \\ Biomedicine and Food Science, \\ University of Padova, Legnaro (PD); \\ ${ }^{2}$ Intitute for Quality and Agrifood \\ Technology, Veneto Agricoltura, Thiene \\ (VI), Italy
}

\section{Abstract}

Challenge tests are a clear opportunity for manufacturers interested in the evaluation of their management system with the aim to reduce the spread of foodborne pathogens. This is a main concern especially in ready-to-eat food in relation to the risk associated with Listeria monocytogenes. For small and medium-scale food industry the manufacturing practices and products formulation are characterised by a wider variability and poor repeatability. The use of ad hoc challenge test and the comparison among different processing systems are strongly required. This paper reports a preliminary comparison among different challenge tests $(n=12)$ commissioned by three manufacturers of raw-fermented salami during a period of three years (2013-2016). The challenge tests were designed to evaluate the growth potential $(\delta)$ of $L$. monocytogenes during the whole processing period of the salami. The doughs were prepared according to different formulations: the simplest formulation was represented by the use of salt, potassium nitrate, black pepper and starter cultures, while the most composited formulations also included the use of sugars and ascorbic acid in addition to nitrite salt. All the processing steps were conducted within an experimental laboratory dedicated for the processing of meat After stuffing, the salami were dried and ripened under temperature and relative humidity control. The sugar inclusion can be considered as a protective factor, while the drying step at high temperature (above $20^{\circ} \mathrm{C}$ ) was associated with higher $\delta$ values $\left(\delta>0.5 \log _{10} \mathrm{cfu} / \mathrm{g}\right)$. The addition of starter cultures, and the subsequent acidification highlighted the importance of $\mathrm{pH}$ as the parameter able to affect the L. monocytogenes growth.

\section{Introduction}

The growth conditions of Listeria monocytogenes are widely described however, in most cases, data are based only on laboratory media. In these conditions, $L$. monocytogenes is able to replicate at activity water $\left(\mathrm{a}_{\mathrm{w}}\right)$ of $0.93, \mathrm{pH} 4.2-4.3$ and salt concentration upper to $12 \%$ (European Union Reference Laboratory for Listeria monocytogenes, 2014).

According to these observations, the physical-chemical parameters of fermented salami can be considered, in many cases, as permissive for the Listeria growth. Due to its psychrotrophic habits, its ability to survive on different surfaces and its resistance to environmental conditions, L. monocytogenes is a serious hazard during all the manufacturing practices regarding the salami production (Nightingale et al., 2006). In comparison to other ready-to-eat (RTE) foods, the physical-chemical variables (e.g. $\mathrm{pH}, \mathrm{a}_{\mathrm{w}}$, salt concentration) of fermented salami change along the ripening period and also during the post-processing conservation. Moreover, small and medium-sized enterprises usually apply a wide range of processing techniques, such as different temperatures of drying, ingredients and preservatives (e.g. nitrites) or specific starter cultures. Taking this into account, challenge tests are required for the validation of process manufacturing and products (Nightingale et al., 2006). Recently, a technical guidance document to determine the shelf life of ready-to-eat products was updated in agreement with L. monocytogenes microbiological criteria fixed by the European legislation (European Union Reference Laboratory for Listeria monocytogenes, 2014). Several microbiological procedures can be applied to evaluate the growth of L. monocytogenes, in naturally contaminated food (durability studies) or in artificially contaminated matrices (challenge tests). In general, for challenge tests two different approaches can be conducted: challenge test that describe if L. monocytogenes is able to grow in a certain food (growth potential) and Challenge tests that elucidate how L. monocytogenes grows in a food in order to establish the maximum growth rate (European Union Reference Laboratory for Listeria monocytogenes, 2014). With the aim to assess the growth boundary for L. monocytogenes, the evaluation of product characteristics is the basis for the robust safety management on ready to eat foods (Mejlholm and Dalgaard, 2007). The development of predictive mod-
Correspondence: Luca Fasolato, Department of Comparative Biomedicine and Food Science, University of Padova, viale dell'Università 16, 35020 Legnaro (PD), Italy. Tel: +39.49.8272965 - Fax: +39.49.827.2604. E-mail: luca.fasolato@unipd.it

Key words: Challenge test; Salami; Listeria monocytogenes; Growth potential.

Conflict of interest: the authors declare no potential conflict of interest.

Received for publication: 20 July 2016. Revision received: 22 December 2016. Accepted for publication: 22 December 2016.

This work is licensed under a Creative Commons Attribution-NonCommercial 4.0 International License (CC BY-NC 4.0).

(C) Copyright E. Novelli et al., 2017

Licensee PAGEPress, Italy

Italian Journal of Food Safety 2017; 6:6184

doi:10.4081/iifs.2017.6184

els that explain the effects of a wide range of food variables (e.g., phenols content, nitrite, $\mathrm{pH}, \mathrm{NaCl}$ ) can elucidate some critical aspects on the food formulae/food process. Moreover, these approaches allow the selection of the most important attributes affecting the growth of pathogens (Mejlholm and Dalgaard 2007; Medina et al., 2016).

Here, an explorative study was conducted with the use of a free on-line web-platform to fit bacterial curves (Combase; http:/www.combase.cc/index.php/en/) in combination with several multivariate statistical analyses (Clarke and Gorley, 2006). The application of classical predictive microbiology together with several approaches applied in the ecology fields could elucidate additional relations among microbial and physicochemical features. This paper elucidates the different behaviors of L. monocytogenes in fermented salami. The study was conducted on a type of salami whose recipe and production methods are based on those of the traditional local production. The results of twelve challenge tests were evaluated during the period of three years (2013-2016).

Several microbial outlines such as $\log _{10}$ $\mathrm{cfu} / \mathrm{g}$, the growth potential $(\delta)$ and the fit model parameters of growth curves were linked with the physical-chemical variables and some manufacturing details with the aim to highlight the critical points of this fermented meat product. 


\section{Materials and Methods}

\section{Salami manufacture}

The meat products object of the study were produced from three different companies who have undertaken the choice of the challenge test with the aim to monitor their manufacturing practices and the conditions of the end product. Therefore, we are referring to microbial challenge tests regarding the technology process.

The meat mixtures were formulated using lean cuts (muscles of the thigh, shoulder and loin) and fats (throat and belly) in a ratio around $80 / 20$. Then, the raw material was ground through molds with holes of 7 or $8 \mathrm{~mm}$. Ground meat was subsequently kneaded with salt (from $2.4 \%$ to $2.7 \%$ ), sugars (sucrose and dextrose), potassium nitrate and sodium nitrite, spices (black pepper). A mix of commercial starter culture is generally used. The dough was stuffed using natural casing, obtaining units of $0.8-1 \mathrm{~kg}$. After a short period of dripping (6-8 hours) conducted at room temperature without any regulation of the humidity, the salami started the drying step first (also called fermentation step) and the ripening period later whose length and conditions of temperature and humidity were those own of each company. The differences in the recipe formulation and in the processing applied by each company, that may have relapses on L. monocytogenes growing, are resumed in the Table 1. Some variables have been categorised with the aim to reduce the small discrepancy such as the sampling times that are overall summarised at $0,7,15,25,30,40$ and 50 days. All the activities of meat processing and subsequent drying and ripening were conducted in a laboratory of the University of Padova fitted for the conduction of challenge tests.

\section{Challenge tests}

Twelve Challenge tests (Table 1) assessing growth potential $(\delta)$ were performed during a period of three years (2013-2016). After the ripening period, the $\delta$ were also evaluated in the post-processing storage when salami were held in different conditions (e.g, temperature), however this data were not considered in this paper. Each test was conducted with a mix of strains of L. monocytogenes: L1 and L2 were isolated from salami produced locally and one reference strain LMG13305. The pre-inoculum of each strain was standardised (Mcfarland $0.5)$ and then diluted $1: 100$. One $\mathrm{mL}$ of each sub-culture was mixed together, then $3 \mathrm{~mL}$ of the mix was added to $100 \mathrm{~mL}$ of sterile skimmed milk $\left(30 \mathrm{~min}\right.$ at $20^{\circ} \mathrm{C}$ ). This volume has been used for the experimental contamination of each batch of dough (added during the kneading step). The most important thing that is shared in the whole test is that the initial contamination of each dough was around $2 \log _{10} \mathrm{cfu} / \mathrm{g}$ in all the experiments.

During the ripening period, three sampling units for each batch were submitted to microbiological analyses. Quantitative analyses were carried out to verify the amount of L. monocytogenes (ISO 11290-2; ISO, 1998).

\section{pH, water activity and weight loss}

The $\mathrm{pH}$ was recorded directly using a Xerolyt Plus penetration electrode (Mettler Toledo, Urdof, Switzerland) assembled on a portable pH-meter (Knick 911; Knick International, Berlin, Germany) coupled with a thermometer probe for temperature compensation. For each sample unit the measure was taken both in the geometrical center and just below the casing. $a_{w}$ measurement was taken with a hygrometer AquaLab 4 TEV (Decagon Devices, Pullman, WA, USA), on a slice of the entire section of each sample unit $(0.5 \mathrm{~cm})$ that was cut into cubes of approximately $5 \mathrm{~mm}$ side that, after manual mixing using a plastic spatula, were placed in three teflon cups sealed with their lid and used for direct measurement. The weight loss (loss \%) was calculated as the percentage difference in weight between the first (just after dripping) and the last day of ripening.

\section{Statistical analysis}

The $\delta$ values were calculated at each sampling time during ripening. This index was the difference between the $\log _{10} \mathrm{cfu} / \mathrm{g}$ at each period of sampling and the $\log _{10} \mathrm{cfu} / \mathrm{g}$ measured in each batch at the first day of processing. When the $\delta$ values were $\leq 0.5$ $\log _{10} \mathrm{cfu} / \mathrm{g}$ during all the ripening period, the products were considered as unable to support L. monocytogenes growth (European Union Reference Laboratory for Listeria monocytogenes, 2014). The $\log _{10}$ $\mathrm{cfu} / \mathrm{g}$ of each challenge tests were modeled with the DMFit program in order to define the following parameters: initial value ( $\left.\log _{10} \mathrm{cfu} / \mathrm{g}\right), \mathrm{Lag} / \mathrm{shoulder}(\mathrm{h})$, maximum rate $\left(\log _{10} \mathrm{cfu} / \mathrm{g} / \mathrm{h}\right)$, final value $\left(\log _{10} \mathrm{cfu} / \mathrm{g}\right)$ (Baranyi and Roberts, 1994; http://browser.combase.cc/DMFit. aspx). Two different matrices of microbial data were created, the first one with the $\delta$ values and the $\log _{10} \mathrm{cfu} / \mathrm{g}$ values according

Table 1. Technological factors of salami manufacturing adopted in twelve challenge tests.

\begin{tabular}{|c|c|c|c|c|c|c|c|c|c|}
\hline Producer & Challenge & Salt (\%) & $\mathrm{NO}_{3}(\mathrm{ppm})$ & $\begin{array}{l}\text { Dough formula } \\
\qquad \mathrm{NO}_{2}(\mathrm{ppm})\end{array}$ & Sugar (\%) & Starter ${ }^{\circ}$ & Drying & $\begin{array}{l}\text { Ripening period } \\
\text { (days) }\end{array}$ & $\delta$ \\
\hline M & 1 & 2.5 & 0 & 0 & 0 & 1 & Hot & $0-50$ & $>0.5$ \\
\hline M & 2 & 2.5 & 150 & 0 & 0 & 1 & Hot & $0-50$ & $>0.5$ \\
\hline M & 3 & 2.5 & 0 & 0 & 0 & 1 & Hot & $30-50$ & $>0.5$ \\
\hline M & 4 & 2.5 & 150 & 0 & 0 & 1 & Hot & $30-50$ & $<0.5$ \\
\hline Z & 5 & 2.4 & 100 & 150 & 0 & 0 & Cold & $0-40$ & $<0.5$ \\
\hline Z & 6 & 2.4 & 100 & 150 & 0.6 & 1 & Cold & $0-50$ & $<0.5$ \\
\hline Z & 7 & 2.4 & 100 & 150 & 0.6 & 2 & Cold & $0-50$ & $<0.5$ \\
\hline Z & 8 & 2.4 & 100 & 150 & 0.6 & 3 & Cold & $0-50$ & $<0.5$ \\
\hline Z & 9 & 2.4 & 100 & 150 & 0 & 0 & Cold & $0-50$ & $>0.5$ \\
\hline Z & 10 & 2.4 & 100 & 150 & 0.6 & 1 & Cold & $0-50$ & $<0.5$ \\
\hline Z & 11 & 2.4 & 100 & 150 & 0.6 & 4 & Cold & $0-50$ & $<0.5$ \\
\hline G & 12 & 2.7 & 100 & 150 & 0.3 & 4 & Cold & $0-40$ & $<0.5$ \\
\hline
\end{tabular}

${ }^{\circ} 0$, no starter; 1, Staphylococcus xylosus + Lactobacillus curvatus; 2, Staphylococcus xylosus +Lactobacillus sakei; 3, Pediococcus pentosaceus + Staphylococcus xylosus; 4 , Staphylococcus xylosus + Lactobacillus cur vatus + Lactobacillus sakei. Italics type highlights the $\delta$ values where the end product was able to support the growth of Listeria monocytogenes. 
to the mean of each triplicate (65 observations-matrix 1), the second one considering only the growth parameters estimated by the Baranyi and Roberts models (12 challenges; 12 observations-matrix 2).

The use of multivariate analysis to evaluate the behaviors of different challenge tests was recently proposed (Medina et al., 2016). Hierarchical cluster analysis and non-metric multi-dimensional scaling plot were first adopted to explore the two matrices; Gower dissimilarity measures were applied to create resemblance matrixes (Anderson et al., 2006).

With the aim to investigate the relationship among physical-chemical variables $\left(\mathrm{a}_{\mathrm{w}}, \mathrm{pH}\right.$ external, $\mathrm{pH}$ internal, loss $\%$ ) and the microbial data (two matrices), distance based redundancy analyses (dbRDA) and distance-based multivariate analyses for a linear model (DISTLM) were performed (Anderson, 2003; Clarke and Gorley, 2006).

One-way permutational multivariate analysis of variance (PERMANOVA) tests were carried out (Anderson, 2001) to highlight the effects of the fixed factors (technological variables: ripening period, starter supplementation, temperature of drying, $\mathrm{NO}_{2}$ and $\mathrm{NO}_{3}$ supplementation, sugar supplementation, salt $\%$ ). In case of significant effects, a posteriori pair-wise comparisons were determined.

The values of $\delta$ were categorised in dichotomous dummy-variables $(0=\leq 0.5$ $\log _{10} \mathrm{cfu} / \mathrm{g}$ and $\left.1=>0.5 \log _{10} \mathrm{cfu} / \mathrm{g}\right)$. The dummy features were applied as dependent variables in several stepwise binary logistic regression models to highlight the best descriptors of the potential of growth. The physical-chemical variables $(\mathrm{pH}$ internal; $\mathrm{pH}$ external; loss $\left.\% ; \mathrm{a}_{\mathrm{w}}\right)$ and technological factors (days of sampling, ripening period, starter supplementation, starter species, $\mathrm{NO}_{3}$ and $\mathrm{NO}_{2}$ supplementation, sugar supplementation, temperature of drying, salt $\%$, company) were tested separately. These analyses were performed using the IBM $^{\circledR}$ SPSS ${ }^{\circledR}$ Statistics 20 Core System.

\section{Results and Discussion}

\section{Challenge tests}

The challenge tests reported in this paper were commissioned by three different manufacturers, therefore, it is plausible that there are differences both in the formulation of the dough as in the process technology. The results that are shown could be considered as a comparison among different processing technologies, therefore, both the discussion as the conclusions may not have general value, but must be ascribed to the processing data indicated in the synoptic table (Table 1). Four challenge tests highlighted some critical issues in the management processes and these had a clear effect showed by salami that were able to support the growth of L. monocytogenes (Table 1 and Figure 1).

The majority of the challenge tests highlighted a decrease of $L$. monocytogenes during the ripening period (Figure 1, blue colour), the challenge tests that pointed out $\delta$ values $>0.5 \log _{10} \mathrm{cfu} / \mathrm{g}$ were characterised by an initial lag phase followed by a positive maximum growth rate and, an higher final $\log _{10} \mathrm{cfu} / \mathrm{g}$. However, due to the heterogeneity of sampling times, these data may be considered only as descriptive. These results suggested similar behaviors among the curves of growth in the case of test that showed $\delta>0.5 \log _{10} \mathrm{cfu} / \mathrm{g}$. For instance, the hierarchical analysis highlighted an 80 and $60 \%$ similarity between the couple of challenges 3-9 and 1-2, respectively.

\section{Physical-chemical data}

The DISTLM and dbRDA analyses highlighted that $\mathrm{pH}$ internal $(\mathrm{P}=0.001)$ and $\mathrm{pH}$ external $(\mathrm{P}=0.021)$ of salami were the most important variables in explaining the variation in the L. monocytogenes data (Figure 2). The binary logistic regression confirmed this observation, the model build with both variables allowed an overall percentage of samples classification equal to $75.4 \%$. The Regulation 2073/2005 (European Commission, 2005) applied a specific threshold of 4.4 for $\mathrm{pH}$ values in

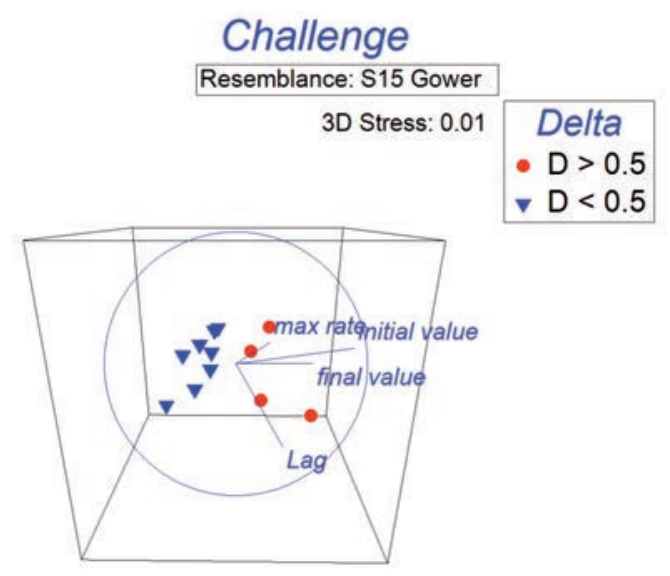

Figure 1. Non-metric multi-dimensional scaling plot of matrix 2 (growth parameters estimated through Baranyi and Roberts models).

\section{L.monocytogenes}

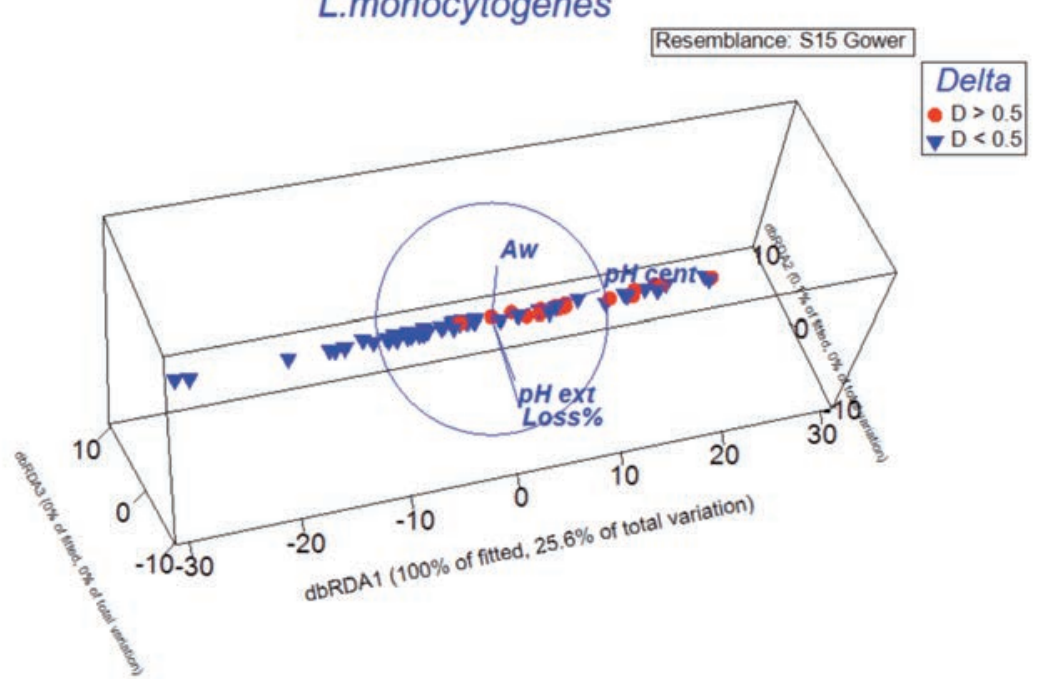

Figure 2. Distance based redundancy analysis of matrix 1 . Matrix 1 was formed by Listeria monocytogenes $\log _{10} \mathrm{cfu} / \mathrm{g}$ and $\delta$ values (response variables); the physicochemical variables were superimposed (predictors). The position of each observation was labelled according to the $\delta$ values $($ red=able to grow $\delta>0.5$, blue=unable to grow $\delta<0.5$ ). 
RTE food, it is assumed that at this of $\mathrm{pH}$ the growth of Listeria monocytogenes is dramatically reduced. However, this limit can be raised if also $a_{w}$ is considered $\left(\mathrm{pH} \leq 5.0\right.$ and $\left.\mathrm{a}_{\mathrm{w}} \leq 0.94\right)$. The levels of $\mathrm{pH}$ were always over the proposed limits in all the series of experiments, the salami that highlighted $\delta>0.5$ showed higher internal $\mathrm{pH}$ ( $\mathrm{pH}$ int values: median 5.4, $\max$ 5.7, min 5.0) if compared to the others products that did not allowed the growth $(\mathrm{pH}$ int values: median 5.1, $\max 5.9 \min 4.6$ ). As reported in Figure 2, the internal $\mathrm{pH}$ was the best predictor of Listeria growth (response variables), the $\mathrm{pH}$ vector suggested that the $\delta>0.5$ samples were ascribed in the cluster with the highest internal $\mathrm{pH}(\mathrm{pH}$ int). Interestingly, the external $\mathrm{pH}$ showed a different vector direction. In general, only few salami showed an $\mathrm{a}_{\mathrm{w}}$ with values unable to support the growth of Listeria (only 4 experiments). The $\mathrm{a}_{\mathrm{w}}$ values decreased under the 0.92 value only after 40 days. According to the thresholds proposed by the Regulation 2073/2005 $\left(\mathrm{pH} \leq 5.0\right.$ and $\mathrm{a}_{\mathrm{w}} \leq$ 0.94 ), the synergic effect among parameters could be reported for other 4 additional challenges tests $\left(\mathrm{a}_{\mathrm{w}}\right.$ median $=0.94$ and $\mathrm{pH}$ median=4.95) (European Commission, 2005).

These data are in agreement with the classical Mediterranean-style fermented sausages where the $\mathrm{pH}$ is around 4.5-5.4, while the values of activity water seemed higher than the threshold of 0.90 proposed for the microbial stability in order to prolong the shelf life of salami (Meloni, 2015; Heinz and Hautzinger, 2007).

In vitro studies on the sub-lethal and lethal effects of $\mathrm{a}_{\mathrm{w}}$ and $\mathrm{pH}$ suggested that $L$. monocytogenes showed a certain acid tolerance respect to the osmotic tolerance (Tigantias et al., 2009). However, the sequential exposure to stressors, as occur during ripening of salami, with an initial acidification followed by the osmotic shift (upshift) induced strong effects in growth and in the adaptation of L. monocytogenes (Tigantias et al., 2009).

In a real production system, these parameters are largely influenced by several technological factors such as the calibers of the products, the drying period and the use of starter cultures (Heinz and Hautzinger, 2007). The temperature adopted during the different technological processes (e.g. drying, ripening, storage) could be considered as a key for the reduction of the risk associated to Listeria (Tigantias et al., 2009).

The present results confirmed that the measure of $\mathrm{pH}$ could be routinely adopted to monitor the successful of each ripening in relation to the behavior of L. monocytogenes.

\section{Importance of technological factors involved in the manufacture}

Figure 3A shows the vectors of each technological factor here considered. The factor identified as temperature of drying was categorised into two dummy variables as cold (0) and hot (1). It must be stressed that when we speak of cold and hot drying temperatures, we refer to the initial 48-72 h, and not the whole length of this step (5-7 days). The samples with $\delta>0.5$ (permissive environment for L. monocytogenes growth) fell in the hot drying condition. On the opposite, the inclusion of sugar into the dough showed a brake in the Listeria growth. The others factors, albeit with different weight, concurred to the regulation in the growth of Listeria. Permanova tests suggested that the most part of the technological factors, with the exception of the ripening periods, affected the microbial growth (matrix 1). The ripening did not affected Listeria, probably because the threshold $\delta>0.5$ in the challenges $1,2,3$ and 9 was reached before the seventh day. The values of $\delta$ and the $\log _{10}$ cfu/g were always above the limit proposed, with a progressive decrease in the final part of the ripening period (after the fifth day).

These data suggested that, regardless the length of ripening, the supplementation with $\mathrm{NO}_{2} \quad(\mathrm{P}=0.0001)$ and/or $\quad \mathrm{NO}_{3}$ $(\mathrm{P}=0.0002)$, the sugar addition $(\mathrm{P}=0.0001)$, the use of starter $(\mathrm{P}=0.0001)$ and the species $(\mathrm{P}=0.0002)$ of starter applied, played a key role in the reduction of the growth potential. Moreover, the cold condition during the drying appears to play a protective effect $(\mathrm{P}=0.0001)$. These preliminary analyses $\mathrm{did}$ not considered the possible interaction among factors, as a consequence of the heterogeneity of the experimental designs.

However, the pair-wise comparisons among the levels of each factor could provide additional speculation. The different species of the starter culture employed showed a different effect on the growth of Listeria. The mix with the species Staphylococcus xylosus and Lactobacillus curvatus influenced the growth of Listeria

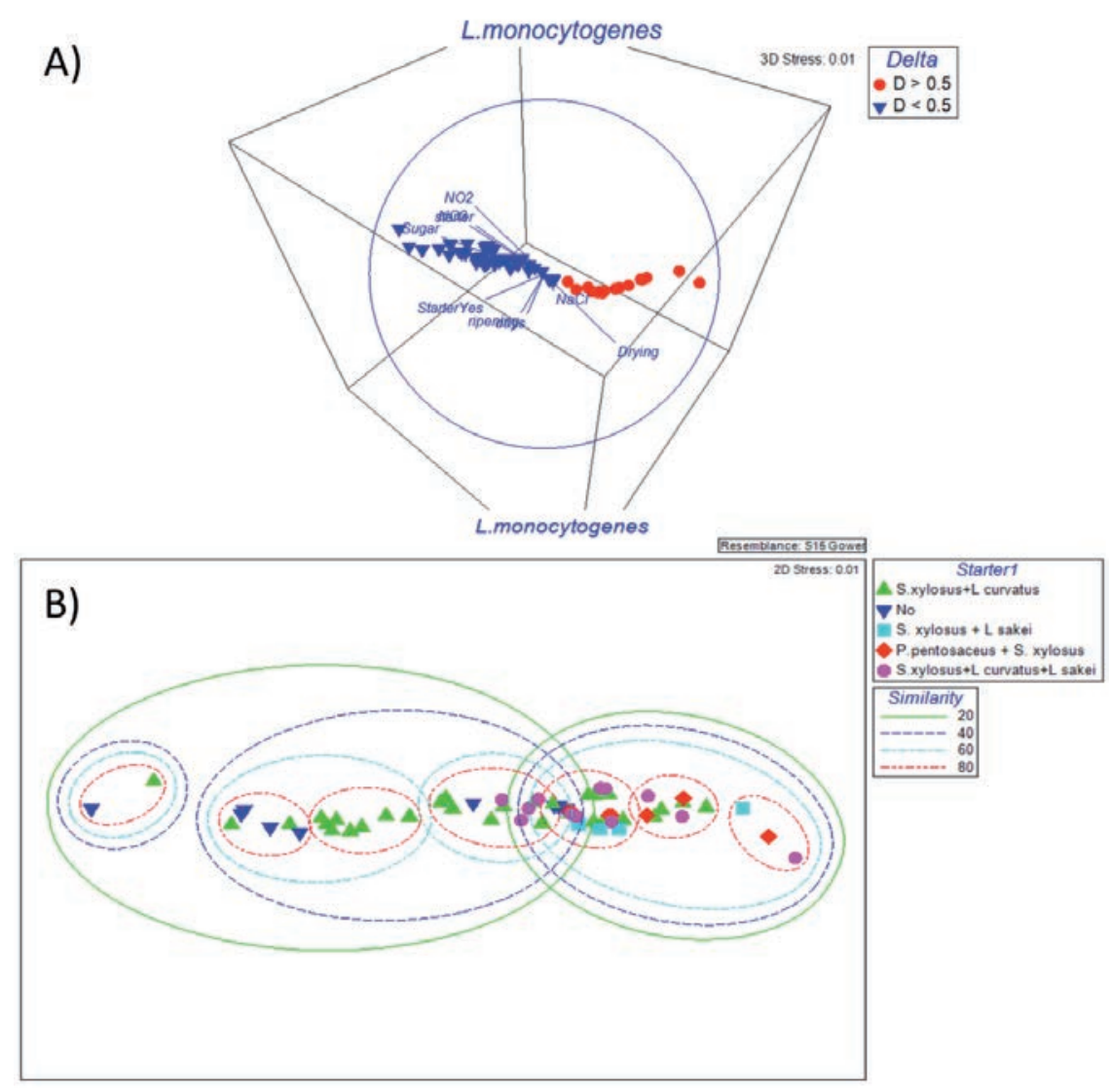

Figure 3. A) Non-metric multi-dimensional scaling plot of matrix 1; the vectors of each studied technological factor was superimposed. The position of each observation was labelled according to the $\delta$ values $($ red $=$ able to grow $\delta>0.5$, blue=unable to grow $\delta<0.5)$. B) Non-metric multi-dimensional scaling plot overlapped with the similarities obtained by hierarchical cluster analysis. 
in a similar way as what observed in the doughs without starter cultures. Figure 3B displayed these differences, where one cluster was composed at the $20 \%$ of similarity including the salami without starter (NO) and the salami with $S$. xylosus + L. curvatus. The other starter cultures were grouped together in a second cluster that was statistically different. It seems that different starters cause different physical-chemical environments, where the salami without starter showed a different pattern on the $\mathrm{pH}$, $\mathrm{a}_{\mathrm{w}}$ and $\operatorname{loss} \%$. Among the mixed cultures the S. xylosus + L. curvatus + L. sakei caused a different physical-chemical environment respect to the others. The effect of lactic acid bacteria in the control of foodborne disease bacteria, in this case L. monocytogenes, is the object of great interest either from the research viewpoint as from the application by the industry. Quinto et al. (2016) studied the dynamics of the growth of L. monocytogenes Scott A cultured alone or co-cultured with a bacteriocin-producing strain Lactobacillus sakei $\mathrm{MN}$ at different inoculum concentrations in a model meat gravy at different temperatures. The inhibitory action of the LAB was clearly evident at each level of temperature teste. The growth's kinetic of $L$. monocytogenes and L. sakei was monitored using the Baranyi growth model evidencing that there was not a direct relationship between the growth rate of L. sakei and its inhibitory effect on L. monocytogenes. Giuffrida et al. (2009) used a stochastic approach with the attempt of modelling the behaviour of $L$. monocytogenes and lactic acid bacteria during the fermentation step of a typical Sicilian salami. Considering that the basic processing parameters, such as temperature, $\mathrm{pH}$ and water activity (stochastic variables), are subjected to continuous variation during the fermentation step, the authors postulated the risk of overestimation of the pathogenic bacteria growth if the competitive microflora, such as LAB among others, are not considered in the microbial modelling. The authors used the Lotka-Volterra model to demonstrate that the real evolution of $L$. monocytogenes during the fermentation step is mainly affected by bacterial competitions which, in turns, is dependent from the intrinsic and extrinsic processing variables. The binary logistic regression selected the Sugar supplementation as a protective factor (model with the $80 \%$ of samples correctly classified; Nagelkerke R Square 0.587 ) that is in agreement with the observation on the $\mathrm{pH}$. This result stressed the importance of a rapid acidification during the first hours of drying.

\section{Conclusions}

The elaboration of microbiological and physical-chemical results achieved through $\mathrm{n}=12$ challenge tests allows to draw, even if with preliminary values, some considerations. The acidification that occurs early in the drying process (fermentation step) appears to play a fundamental role on the ability to limit the growth of L. monocytogenes. However, $\mathrm{pH}$ is only a synthetic indicator of a combined effect due to some microbial species added to the mixture as starter cultures in the presence of adequate amounts of rapidly fermentable sugars. It must be stressed the need to improve the knowledge about the inhibitory effect done by the competitive microflora: just the acidification of the matrix or also the spread of the bacteriocines in the neighbourhood? In parallel, it is noteworthy the importance of modelling the behaviour of L. monocytogenes with respect to the dynamic of the physical-chemical variables that govern the fermentation phase. It is interesting to find an apparently inhibiting effect attributable to the conduction of the initial step of drying at low temperature. The latter is an output that is worth of further studies. The length of the ripening step did not seem have any effect on L. monocytogenes, as was the addition of nitrate and nitrite salts.

\section{References}

Anderson MJ, 2001. A new method for nonparametric multivariate analysis of variance. Austral Ecol 26:32-46.

Anderson MJ, 2003. DISTLM forward: a FORTRAN computer program to calculate a distance-based multivariate analysis for a linear model using forward selection. Department of Statistics, University of Auckland, New Zealand.

Anderson MJ, Ellingsen KE, McArdle BH, 2006. Multivariate dispersion as measure of beta diversity. Ecol Lett 9:68393.

Baranyi J, Roberts TA, 1994. A dynamic approach to predicting bacterial growth in food. Int J Food Microbiol 23:27794.

Clarke KR, Gorley RN, 2006. PRIMER V6: user manual and tutorial. PRIMER-E Ltd, Plymouth, UK.

European Commission, 2005. Commission Regulation of 15 November 2005 on microbiological criteria for foodstuffs, 2073/2005/EC. In: Official Journal, L 338/1, 22.12.2005.

European Union Reference Laboratory for
Listeria monocytogenes, 2014. EURL Lm technical guidance document for conducting shelf-life studies on Listeria monocytogenes in ready-to-eat foods Version 3, 6 June 2014. Available from: EURL Lm_Technical Guidance Document $\quad$ Lm shelf-life studies_V3_2014-06-06.pdf

Giuffrida A, Valenti D, Ziino G, Spagnolo B, Panebianco A, 2009. A stochastic interspecific competition model to predict the behaviour of Listeria monocytogenes in the fermentation process of a traditional Sicilian salami. Eur Food Res Technol 228:767-75.

Heinz G, Hautzinger P, 2007. Meat processing technology for small-to medium scale producers, chapter: raw-fermented sausages. Available from: http:/www.fao.org/docrep/010/ai407e/ ai407e00.htm

ISO, 1998. Microbiology of food and animal feeding stuffs. Horizontal method for the detection and enumeration of Listeria monocytogenes. Part 2: Enumeration method. ISO Norm 11290-2:1998. International Organization for Standardization, Geneva, Switzerland.

Medina E, Romero-Gil V, GarridoFernández A, Arroyo-López FN, 2016. Survival of foodborne pathogens in natural cracked olive brines. Food Microbiol 59:104-11.

Mejlholm O, Dalgaard P, 2007. Modeling and predicting the growth boundary of Listeria monocytogenes in lightly preserved seafood. J Food Protect 70:7084.

Meloni D, 2015. Presence of Listeria monocytogenes in Mediterranean-style dry fermented sausages. Foods 4:34-50.

Nightingale KK, Thippareddi H, Phebus RK, Marsden JL, Nutsch AL, 2006. Validation of a traditional Italian-style salami manufacturing process for control of Salmonella and Listeria monocytogenes. J Food Protect 69:794-800.

Quinto EJ, Marín JM, Schaffner DW, 2016. Effect of the competitive growth of Lactobacillus sakei $\mathrm{MN}$ on the growth kinetics of Listeria monocytogenes Scott A in model meat gravy. Food Control 63:34-45.

Tiganitas A, Zeaki N, Gounadaki AS, Drosinos EH, Skandamis PN, 2009. Study of the effect of lethal and sublethal $\mathrm{pH}$ and aw stresses on the inactivation or growth of Listeria monocytogenes and Salmonella Typhimurium. Int J Food Microbiol 134:104-12. 